\title{
Test Strip Device
}

National Cancer Institute

\section{Source}

National Cancer Institute. Test Strip Device. NCI Thesaurus. Code C50322.

A piece of chemically treated medium designed to react in the presence of specific amounts of a trigger substance. 